\title{
Energy flux of electromagnetic field in stochastic model of radiative heat transfer in dielectric solid medium
}

\author{
Yu.P. Virchenko, Lam Tan Phat \\ Belgorod State University, 308015, Belgorod, Russia \\ Received October 31, 2016
}

\begin{abstract}
The stochastic model that describes radiative heat transfer in dielectric medium is analyzed. The model is based on the representation that heat transfer is realized both by its heat conductivity and by electromagnetic radiation that is generated by thermal fluctuations in the medium. Such physical suppositions, using fluctuation-dissipative theorem, are realized in the form of the infinite dimensional Ornstein-Uhlenbeck process that describes medium fluctuations. In the model frameworks, the energy flux density of fluctuating electromagnetic field is calculated in the form of a functional of the temperature distribution in real three-dimensional media sample.

Keywords: radiative heat transfer, fluctuation-dissipative theorem, infinite dimensional Ornstein-Uhlenbeck process.
\end{abstract}

Анализируется стохастическая модель радиационно-кондуктивного теплообмена в твердотельной диәлектрической среде. Модель основана на представлении о переносе тепла, наряду с собственной теплопроводностью среды, также посредством теплового әлектромагнитного излучения, которое порождается тепловыми флуктуациями. Эти физические представления, используя флуктуацинно-диссипадионную теорему, реализованы в виде бесконечномерного процесса Орнштейна-Уленбека, описывающего флуктуации среды. В рамках модели вычислен поле плотности потока энергии флуктуационного электромагнитного поля в реальном трехмерном образце среды в виде функционала от распределения температуры.

Струм енергії електромагнітного поля у стохастично моделі радіаційно-кондуктивного теплообміну у діелектричному твердотільному середовищі. Ю.П. Вірченко, Лал Тан Фат.

Аналізуеться стохастична модель радіаційно-кондуктивного теплообміну у твердотільному діелектричному середовищі. Модель утворена на підставі уявлення о перенесенні тепла, поряд з власної теплопровідністю, також внаслідок теплового электромагнітного випромінювання, що породжуєся тепловими флуктуаціями. Ці фізичні уявлення реалізовані, за допомогою флуктуаційно-дісиапативної теореми, у вигляді нескінченновимірного процесу Орнштейна-Уленбека, що описуе флуктуапії у середовищі. У рамках моделі рахується поле рідини струма енергії флуктуаційного әлектромагнітного поля у реальному трьохвимірному середовищі у вигляді функциіоналу від розподілу температури.

\section{Introduction.}

The heat transfer in solids is realized by two mechanisms. They are the thermal conductivity and the heat transfer by electro-magnetic radiation. The last is generated by thermal fluctuations of medium local thermodynamic state. In accordance with this, the evolution equation of the temperature distribution $T(\mathbf{x}, t)$ at fixed time moment $t$ may be written phenomenologically in the form (see, for example, [1]-[3])

$$
\kappa \dot{T}(\mathbf{x}, t)=\varkappa \Delta T(\mathbf{x}, t)-(\nabla, \mathbf{S})(\mathbf{x}, t)
$$


where $\varkappa>0$ is the medium conductivity coefficient and $\kappa$ is its volume heat capacity. We assume that these quantities do not depend on temperature. The vector field $\mathbf{S}(\mathbf{x}, t)$ is the energy flux density of electromagnetic radiation associated with fluctuations of charges and currents induced by them. The value of $(\nabla, \mathbf{S}(\mathbf{x}, t))$ multiplied by the small volume of the spatial medium region centered near the point $\mathbf{x}$ is the flux part which is spent on its heating at the time moment $t$. Inclusion of this term is essential when heat transfer problems are solved in optically transparent media with low electrical conductivity and at sufficiently large temperature drops on character distances. In this case, it is necessary to build a closed evolution equation for $T(\mathbf{x}, t)$ in order to solve the heat transfer problems. So, it is necessary to find the explicit form of the functional $\mathbf{S}(\mathbf{x}, t)=\mathbf{S}[T(\mathbf{x}, t)]$ which transforms Eq. (1) into a self-consistent one.

Usually, the energy flux density $\mathbf{S}(\mathbf{x}, t)$ is constructed phenomenologically, in frameworks of so-called theory of radiation transfer which is based on: geometrical optics concepts applying them to heat radiation inside the medium, on the Kirchhoff law about the relationship of irradiation and absorption intensities of electromagnetic field and on the Bouguer law (see, for example, [1]-[4]). But thermal electromagnetic field in such theoretical building does not exist. Such a situation exists due to the lack of a consistent microscopic theory of radiation-conductive heat transfer which should be based on the quantum theory of radiation and absorption of thermal photons in solid medium.

We shall not concentrate on a detailed analysis of the problems which are related with the construction of the radiation transfer microscopic theory in the framework of statistical physics (see, for more information, [5]-[7]). We point out only that there is a statistical approach that occupies an intermediate position in the radiation transfer theory between the microscopic and phenomenological ones. It is based on the concept of thermodynamic fluctuations in medium which generate a stochastic electromagnetic field (see, [8]-[9]) without specifying the microscopic transition mechanism of its energy into heat. This field is caused by thermal fluctuations of charges and fluctuations of electrical currents induced by them. The currents at short distances appear in the medium even if it has very low electrical conductivity. The amplitude of these fluctuations increases on temperature, so, at sufficiently large its value, thermal vibrations of medium atoms (ions) leads to fluctuations of electrical charges on those spatial scales which are comparable with the interatomic ones.

At present work we analyze, within the mentioned fluctuation approach, the stochastic model thermal radiation transfer proposed in [10] which is based on explicit statistical description of thermal fluctuations of charges and currents in the medium. Due to complexity of used mathematical constructions, we focus our considerations only on the case when the inhomogeneity of temperature distribution is concentrated in a limited region of boundless environment.

\section{The model construction}

Thermal electromagnetic field is described by a pair $\{\tilde{\mathbf{E}}(\mathbf{x}, t), \tilde{\mathbf{H}}(\mathbf{x}, t)\}$ at each space-time point $\{\mathbf{x}, t\}$, is stochastic. Then its energy flux density is the random function

$$
\tilde{\mathbf{S}}(\mathbf{x}, t)=\frac{c}{4 \pi}[\tilde{\mathbf{E}}, \tilde{\mathbf{H}}](\mathbf{x}, t),
$$

$c$ is the vaccum light velocity. This field substantially varies over distances having the order of thermal radiation characteristic wavelength $\left(\sim 10^{-4} \mathrm{~cm}\right)$ and during time periods $\sim 10^{-14} \mathrm{sec}$. At the same time, the characteristic length of thermal conductivity process in crystalline dielectrics has the order $10^{-2} \mathrm{~cm}$, and the correspondent characteristic time is $10^{-1} \mathrm{sec}$. Therefore, the vector field (2) should be averaged over spatial regions having a scale which is much greater than the characteristic wavelength, but it is much smaller than the characteristic length of the heat transfer process. In addition, it should be averaged over time intervals which are much greater than the characteristic period of thermal radiation oscillations, but it is much smaller than the characteristic time of thermal conductivity. Such an averaging allows to ignore the small rapid changes of the divergence $(\nabla, \tilde{\mathbf{S}}(\mathbf{x}, t))$ over space and time, which have no a relation to the heat transfer process. The pointed out space-time averaging is equivalent to the averaging using probability distribution of random electromagnetic field, when the pair random fields $\tilde{\mathbf{E}}(\mathbf{x}, t)$ and $\tilde{\mathbf{H}}(\mathbf{x}, t)$ possess the ergodicity property. Thus, the energy flux density of the field used in (1) is determined by 
the mathematical expectation $\mathbf{S}(\mathbf{x}, t)=\langle\tilde{\mathbf{S}}(\mathbf{x}, t)\rangle$. It should be calculated in the frameworks of the model that describes the heat transfer by radiation.

The stochastic electromagnetic field is seemed by random realizations $\{\tilde{\mathbf{E}}(\mathbf{x}, t), \tilde{\mathbf{H}}(\mathbf{x}, t)\}$ which satisfy the system of stochastic Maxwell equations in the continuous dielectric medium neglecting its variance

$$
\begin{array}{ll}
\frac{\varepsilon}{c} \frac{\partial \tilde{\mathbf{E}}}{\partial t}+\frac{4 \pi}{c} \tilde{\mathbf{j}}=[\nabla, \tilde{\mathbf{H}}], & (\nabla, \tilde{\mathbf{E}})=\frac{4 \pi}{\varepsilon} \tilde{\rho}, \\
\frac{\mu}{c} \frac{\partial \tilde{\mathbf{H}}}{\partial t}=-[\nabla, \tilde{\mathbf{E}}], \quad(\nabla, \tilde{\mathbf{H}})=0,
\end{array}
$$

where $\tilde{\mathbf{E}}$ and $\tilde{\mathbf{H}}$ are intensities of electric and magnetic fields, correspondingly. At the same time, $\varepsilon$ is the electric permeability of uniform dielectric medium and $\mu$ is the magnetic one. We consider them to be independent of $\{\mathbf{x}, t\}$.

The values $\varepsilon$ and $\mu$, generally speaking, depend on the temperature. This dependence may be substantial at large temperature drops when distances between spatial points have the order of the medium sample size. Therefore, it is necessary to take into account in problems of heat transfer by radiation when such drops are present. It is supposed that temperatures are equal to the local temperature $T(\mathrm{x}, t)$ of the value order in these dependencies. In general case, spatial and temporal derivatives of $\varepsilon(T(\mathbf{x}, t))$ and $\mu(T(\mathrm{x}, t))$ should be appeared in the Maxwell equations when these dependencies are taken into account. However, these derivatives are extremely small in comparence with those length and time scales which are characteristic of the thermal radiation by virtue of these slow $T(\mathbf{x}, t)$ dependence. Therefore, these derivatives are not accounted in the equations (3).

Solutions of the system (1) are determined by setting of stochastic sources $\tilde{\mathbf{j}}, \tilde{\rho}$ which are the electric current and charge densities, respectively, that are nonzero in micro-regions having the order of the characteristic wavelength. Besides, for complete determination of the solutions, initial and field boundary conditions corresponding to physical situation under consideration are important. As for boundary conditions, we shall study the simplest physical situation in present work. It consists of the attenuation of the localized thermal non-uniformity in unbounded medium. This non-uniformity is concentrated in a bounded space area with the linear size $L$ having the order of $1 \mathrm{~cm} \div 10^{2} \mathrm{~cm}$. In such a situation, the medium temperature tends to a constant value when the point $\mathbf{x}$ goes to infinity. More complicated physical situation from the boundary conditions viewpoint has been investigated in [5]-[7] on the basis of more simple stochastic model.

Densities $\tilde{\mathbf{j}}, \tilde{\rho}$ which are spatially distributed stochastic sources, define completely the model. At the same time, they should be submitted to the continuity equation

$$
\dot{\tilde{\rho}}+(\nabla, \tilde{\mathbf{j}})=0 .
$$

For this reason, the model is completely determined by the form of the stochastic field $\tilde{\mathbf{j}}(\mathbf{x}, t)$. The current density $\tilde{\mathbf{j}}$ is composed of the internal «electromotive force» of the medium that arises as a result of the thermal fluctuations. It should be contained the term which is determined by Ohm's law $\sigma \tilde{\mathbf{E}}$. At the same time, we note that the coefficient $\sigma>0$ which plays the role of electrical conductivity, is not connected with genuine macroscopic electrical conductivity of the medium that may be very small in the physical situation under consideration. It performs an «effective conductivity» which should be different from zero due to the so-called fluctuation-dissipative theorem (see, for example, [9]). It is necessary to account from the mathematical viewpoint in order that a regular dissipative constituent is present in the system of stochastic evolution equations (1) with additive noise. In turn, it is connected with necessity of stationary evolution regime presence.

The part of the fluctuation current density $a(\mathbf{x}, t ; T) \tilde{\varphi}$ that serves stochastic source of electromagnetic field should be certainly contained with probability one the vortical term (fluctuation «Foucault current») in spite of the radiation transfer occurs in a dielectrics (or high-resistance semiconductor). Here, the source intensity $a(\mathbf{x}, t ; T)$ depends functionally on the local temperature $T=T(\mathbf{x}, t)$. Therefore, it may be changed spatially and temporally. This changing is much slower in comparison with the change of the thermal electromagnetic field. The irradiation of electromagnetic waves which transfer of heat is 
namely associated with the availability of the vortical part. In connection with the dielectric character of medium, the fluctuation current (its correlation function) is concentrated at small space scale that has the order of interatomic distance. Thus, the current density $\tilde{\mathbf{j}}$ should be replaced in equations (3) and (4) by $\tilde{\mathbf{j}}(\mathbf{x}, t)=\tilde{\varphi}(\mathbf{x}, t) a(\mathbf{x}, t ; T)+\sigma \tilde{\mathbf{E}}(\mathbf{x}, t)$ where the intensity $a(\mathbf{x}, t ; T)$ should be defined on the basis of statistical physical consideration for completion of the model construction. We suppose that the squared intensity is determined by thermal photons irradiation in a small spatial area which concentrates near the point $\mathrm{x}$ at the moment time $t$. Therefore,

$$
a^{2}(\mathbf{x}, t ; T)=\hbar \int_{-\infty}^{\infty} \omega^{3} f\left(\frac{\hbar \omega}{\mathrm{k} T(\mathbf{x}, t)}\right) d \omega,
$$

where $f$ is the distribution function of radiation photons frequencies $\omega$. It depends on the temperature $T(\mathbf{x}, t)$ distribution. In this case, we have $a^{2}(\mathbf{x}, t ; T) \sim T^{4}(\mathbf{x}, t)$, when it is the Planck function. As the result of substitution of the explicit form of $\tilde{j}(\mathbf{x}, t)$ into the Eq. (3), we obtain the stochastic equations system with the additive noise $\tilde{\varphi}$ where the field $\tilde{\mathbf{E}}(\mathbf{x}, t)$ is determined by the equation

$$
\frac{\partial \tilde{\mathbf{E}}}{\partial t}+\gamma \tilde{\mathbf{E}}+\frac{4 \pi}{\varepsilon} a \tilde{\varphi}=\frac{c}{\varepsilon}[\nabla, \tilde{\mathbf{H}}], \quad \gamma=\frac{4 \pi \sigma}{\varepsilon},
$$

and the evolution equation of the charge density has the form

$$
\dot{\tilde{\rho}}+\gamma \tilde{\rho}+(\nabla, a \tilde{\varphi})=0
$$

where, as above, we have neglected spatial derivatives of temperature distribution. In general case, the coefficient $\sigma$ depends on the local temperature which changes slowly on $\mathbf{x}$ and $t$. But we neglect this dependence for reasons above pointed out.

The random field $\tilde{\varphi}$ in Eqs. $(6),(7)$ is Gaussian with zero average value $\langle\tilde{\varphi}(\mathbf{x}, t)\rangle=0$ due to supposed physical smallness of thermal fluctuations. At the same time, $\langle\tilde{\rho}(\mathbf{x}, t)\rangle=0$. Then the Gaussian field $\tilde{\varphi}(\mathbf{x}, t)$ is completely determined by the pair correlation function $K_{j_{1} j_{2}}\left(\mathbf{x}_{1}, t_{1} ; \mathbf{x}_{2}, t_{2}\right)=$ $\left\langle\tilde{\varphi}_{j_{1}}\left(\mathbf{x}_{1}, t_{1}\right) \tilde{\varphi}_{j_{2}}\left(\mathbf{x}_{2}, t_{2}\right)\right\rangle$. For physical reasons, the random field $\tilde{\varphi}(\mathbf{x}, t)$ is translational invariant on $\mathbf{x}$ in stochastical sense and it is stationary on $t$ in the sense of the theory random processes. Besides, we assume that this field is stochastically isotropic and temporally reversible. So, its correlation function is represented in the form

$$
K_{j_{1} j_{2}}\left(\mathbf{x}_{1}, t_{1} ; \mathbf{x}_{2}, t_{2}\right)=K\left(\left|\mathbf{x}_{1}-\mathbf{x}_{2}\right|,\left|t_{1}-t_{2}\right|\right) \delta_{j_{1}, j_{2}}
$$

In this case, the source $a \tilde{\varphi}(\mathbf{x}, t)$ of thermal radiation is uniform on $\mathbf{x}$, it is stationary on $t$ and it is stochastically isotropic along directions in Eqs. (6),(7) if we neglect the pointed out slow dependence on the local temperature $T(\mathbf{x}, t)$.

Further, we use some supplement assumptions about properties of the function $K(r, s), r, s>0$. These properties are associated with the locality of correlation functions $K_{j_{1} j_{2}}\left(\mathbf{x}_{1}, t_{1} ; \mathbf{x}_{2}, t_{2}\right)$. For physical consideration, the random field $\tilde{\varphi}(\mathbf{x}, t)$ should have extreme small correlation time. Such correlations should be disappear during the temporal interval equal to several periods of stochastic electromagnetic field oscillations. Then we suppose that $K(r, s) \sim \delta(s)$. In this case, the field $\tilde{\varphi}(\mathbf{x}, t)$ is transformed to a generalized random Gaussian field of the «white noise» type on the temporal variable. Correlations of the field $\tilde{\varphi}(\mathbf{x}, t)$ values are also short-ranged. They disappear at the distance equal to some interatomic lengthes. So, the correlation length is the smallest parameter of linear size dimension in the problem under study. However, for the reasons that will become clear from subsequent analysis, we may not assume that the function $K(r, s)$ is proportional to $\delta(r)$ by the analogy with the temporal variable. So, we use the next representation

$$
K\left(\left|\mathbf{x}_{1}-\mathbf{x}_{2}\right|,\left|t_{1}-t_{2}\right|\right)=K\left(\left|\mathbf{x}_{1}-\mathbf{x}_{2}\right|\right) \delta\left(t_{1}-t_{2}\right),
$$

where the function $K(r)$ is absolutely integrable $\int_{\mathbb{R}^{3}}|K(\mathbf{x})| d \mathbf{x}<\infty$ and it is localized in the zero neighborhood having the order of $r_{0}$, that is $K(r)=r_{0}^{-3} Q\left(r^{2} / 2 r_{0}^{2}\right)$ where $r_{0}>0$ is a small parameter and 
$K=\int_{0}^{\infty} Q\left(\xi^{2} / 2\right) d \xi<\infty$. Here the function $Q(r)$ is concentrated in the region with the linear size of order 1 .

After setting of the random process $\tilde{\mathbf{j}}(\mathrm{x}, t)$ in the stochastic differential equations system (3), the fluctuation electromagnetic field is completely defined by the requirement of its temporal stationarity. At the same time, the random function $\tilde{S}(\mathbf{x}, t)$ is a functional on $T(\mathbf{x}, t)$, and its mathematical expectation

$$
\langle\tilde{\mathbf{S}}(\mathbf{x}, t)\rangle=\frac{c}{4 \pi}\langle[\tilde{\mathbf{E}}, \tilde{\mathbf{H}}](\mathbf{x}, t)\rangle
$$

is determined by the probability distribution of the fluctuation field $\tilde{\varphi}$.

\section{Small parameters}

Consistent mathematical analysis of the stationary random process which has been built in the previous section is very complicated. In particular, in the resulting formula of $S_{j}(\mathbf{x}, t)$ leads to complicated expressions which are inconvenient for practical application. The significant simplification of these expressions is reached when the small parameters are taken into account. The natural problem formulation consist of the calculation of the $S_{j}(\mathrm{x}, t)$ expression as the form of main asymptotic term when these small parameters tend to zero.

Let $L$ be the linear size of temperature non-uniformity that equals to the linear size of region where the significant varying of the temperature $T(\mathbf{x}, 0)$ distribution takes place at the initial moment. We note that the characteristic time during which the temperature distribution change is occurred due to the heat conductivity process, is significantly longer than the time $L / \bar{c}$ during which the thermal electromagnetic radiation overcomes the distance $L$ and goes out of the non-uniformity region (during $\sim 3 \cdot 10^{-13}$ sec when $L \sim 10^{-2} \mathrm{~cm}$ ) where the heat transfer processes occurs. The radiation does not effect on the heat transfer process when it comes out of the system. The natural time for the heat transfer process is determined by the value $L^{2} \kappa / \varkappa$ where the ratio $\varkappa / \kappa$ has the order of $10^{-3} \mathrm{~cm}^{2} / \mathrm{sec}$ in the typical physical situation in solid high-resistance semiconductor crystal. Therefore, the characteristic time of thermal conductivity process is equal to $10^{-1}$ sec. As a result, we obtain the small parameter $(L / \bar{c}) /\left(L^{2} \kappa / \varkappa\right)=\varkappa / L \bar{c} \kappa \ll 1$ having the order of $3 \cdot 10^{-12}$ where $\bar{c}^{2}=c^{2} / \varepsilon \mu$.

Further, we assume that the medium is so semi-transparent that the characteristic distance of the radiation damping is much larger than the above-introduced size $L$. In this case, if we use typical values of specific electrical conductivity, the parameter $\gamma L / \bar{c}$ in dielectrics has the values in the range $3 \cdot\left(10^{-4} \div 10^{-17}\right) \ll 1$ where $\gamma=4 \pi \sigma / \varepsilon$ has the order of $10^{6} \div 10^{-7} \mathrm{sec}^{-1}$. For some semiconductors, the parameter $\gamma L / \bar{c}$ changes in the range $4 \cdot\left(10^{-4} \div 10^{5}\right)$.

In view of the value $r_{0}$ smallness, there is the natural small parameter $r_{0} / L$, since $r_{0} \sim 10^{-8} \mathrm{~cm}$, $L \sim 10 \mathrm{~cm}$ and, therefore, $r_{0} / L \sim 10^{-9}$. Thus, we conclude that the following relations $\varkappa / L \bar{c} \kappa \ll \gamma L / \bar{c} \ll$ $r_{0} / L$ are fulfilled in the case of dielectrics on basis of the mentioned typical values of model parameters. As we can see from the above estimations, the parameter $\gamma L / \bar{c}$ is not small for semiconductors in general case. Thus, the calculation of the energy flux density of the fluctuation electromagnetic field suggested in this work is valid for dielectrics and high-resistance semiconductors.

Since asymptotic term is calculated on many parameters, so, it is needed the refinement of the order of transitions to zero limit. We assume that these transitions are understood as repeated in concordance with the order of their typical physical values. The transition to the limit $r_{0} / L \rightarrow 0$ will be done at the final calculation stage.

\section{Characteristics of stationary process.}

In view of the typical time of thermal conductivity is the biggest parameter of temporal size, then the initial conditions of the fields $\tilde{\mathbf{E}}(\mathbf{k}, t), \tilde{\mathbf{H}}(\mathbf{k}, t)$ and $\tilde{\bar{\rho}}(\mathbf{k}, t)$ become inessential after passing the temporal period which is longer than the time $\kappa L^{2} / \varkappa$. 
Since the field $\tilde{\varphi}(\mathbf{x}, t)$ is stationary temporally, then we may neglect the time dependence of the temperature distribution $T(\mathbf{x}, t)$ in the amplitude $a(\mathbf{x}, t ; T)$ when we use the transition to the asymptotic region $t \gg \kappa L^{2} / \varkappa \sim 10^{-7}$ sec. Therefore, we may do such a neglect in the sources $\tilde{\mathbf{j}}(\mathbf{x}, t), \tilde{\rho}(\mathbf{x}, t)$. In this case, the stochastic fields $\{\tilde{\overline{\mathbf{E}}}(\mathbf{x}, t), \tilde{\mathbf{H}}(\mathbf{x}, t)\}$ may also be considered as stationary ones. In such a situation, it is natural to go to some equations which control the evolution of the amplitudes defined by the fields spectral expansion. They are some generalized functions of $\mathbf{k}$ and $\omega$,

$$
\begin{gathered}
\tilde{\mathbf{E}}(\mathbf{x}, t)=\int_{\mathbb{R}^{3}} \exp [i(\mathbf{k}, \mathbf{x})] d \mathbf{x} \int_{-\infty}^{\infty} \tilde{\mathcal{E}}(\mathbf{k}, \omega) e^{i \omega t} d \omega, \tilde{\mathbf{H}}(\mathbf{x}, t)=\int_{\mathbb{R}^{3}} \exp [i(\mathbf{k}, \mathbf{x})] d \mathbf{x} \int_{-\infty}^{\infty} \tilde{\mathcal{H}}(\mathbf{k}, \omega) e^{i \omega t} d \omega \\
a(\mathbf{x}, t ; T) \tilde{\boldsymbol{\varphi}}(\mathbf{x}, t)=\int_{\mathbb{R}^{3}} \exp [i(\mathbf{k}, \mathbf{x})] d \mathbf{x} \int_{-\infty}^{\infty} \tilde{\iota}(\mathbf{k}, \omega) e^{i \omega t} d \omega \\
\tilde{\rho}(\mathbf{x}, t)=\int_{\mathbb{R}^{3}} \exp [i(\mathbf{k}, \mathbf{x})] d \mathbf{x} \int_{-\infty}^{\infty} \tilde{\varrho}(\mathbf{k}, \omega) e^{i \omega t} d \omega
\end{gathered}
$$

where the generalized random fields $\tilde{\boldsymbol{\iota}}(\mathbf{k}, \omega), \tilde{\varrho}(\mathbf{k}, \omega)$ are complex-valued Gaussian random ones due to Gaussian property of the field $\tilde{\varphi}(\mathbf{x}, t)$, and they have zero average values $\langle\tilde{\boldsymbol{\iota}}(\mathbf{k}, \omega)\rangle=0,\langle\tilde{\varrho}(\mathbf{k}, \omega)\rangle=0$.

In view of the field $a(\mathbf{x}, t ; T) \tilde{\varphi}(\mathbf{x}, t)$ is real, the field $\tilde{\iota}(\mathbf{k}, \omega)$ realizations has the following property $\tilde{\boldsymbol{\imath}}^{*}(\mathbf{k}, \omega)=\tilde{\boldsymbol{\imath}}(-\mathbf{k},-\omega)$ with probability one. Therefore, this field is completely characterized by the correlation function $\bar{K}_{l l^{\prime}}\left(\mathbf{k}, \omega, \mathbf{k}^{\prime}, \omega^{\prime}\right)$ which is positively definite matrix-function on $\mathbf{k} \in \mathbb{R}^{3}$ and $\omega$. It is connected with the correlation function $K_{l l^{\prime}}\left(\mathbf{x}, t ; \mathbf{x}^{\prime}, t^{\prime}\right)$ by the following way

$$
\begin{gathered}
\bar{K}_{l l^{\prime}}\left(\mathbf{k}, \omega, \mathbf{k}^{\prime}, \omega^{\prime}\right)=\delta_{l l^{\prime}} \bar{K}\left(\mathbf{k}, \mathbf{k}^{\prime}\right) \delta\left(\omega^{\prime}-\omega\right) \\
\bar{K}\left(\mathbf{k}, \mathbf{k}^{\prime}\right)=\frac{1}{(2 \pi)^{6}} \int_{\mathbb{R}^{6}} \exp \left[i\left(\left(\mathbf{k}^{\prime}, \mathbf{x}^{\prime}\right)-(\mathbf{k}, \mathbf{x})\right)\right] K\left(\left|\mathbf{x}-\mathbf{x}^{\prime}\right|\right) d \mathbf{x} d \mathbf{x}^{\prime}
\end{gathered}
$$

taking into account the properties of the field $\tilde{\varphi}(\mathbf{x}, t)$ : the stochastic uniformity on $\mathbf{x}$, the stationarity on $t$ and its stochastic isotropy.

Substituting these expansions to Eqs. (3) and (6) and using the uniqueness of Fourier images, we obtain the completely equations system:

$$
\begin{gathered}
i \omega \tilde{\mathcal{E}}(\mathbf{k}, \omega)+\gamma \tilde{\mathcal{E}}(\mathbf{k}, \omega)+\frac{4 \pi}{\varepsilon} \tilde{\imath}(\mathbf{k}, \omega)=\frac{i c}{\varepsilon}[\mathbf{k}, \tilde{\mathcal{H}}(\mathbf{k}, \omega)], \\
\tilde{\mathcal{H}}(\mathbf{k}, \omega)=-\frac{c}{\mu \omega}[\mathbf{k}, \tilde{\mathcal{E}}(\mathbf{k}, \omega)], \quad(\mathbf{k}, \tilde{\mathcal{E}}(\mathbf{k}, \omega))=-\frac{4 \pi i}{\varepsilon} \tilde{\varrho}(\mathbf{k}, \omega), \quad(\mathbf{k}, \tilde{\mathcal{H}}(\mathbf{k}, \omega))=0, \\
i \omega \tilde{\varrho}(\mathbf{k}, \omega)+\gamma \tilde{\varrho}(\mathbf{k}, \omega)+i(\mathbf{k}, \tilde{\iota}(\mathbf{k}, \omega))=0,
\end{gathered}
$$

The solutions of the equations system defined Fourier images $\tilde{\mathcal{E}}(\mathbf{k}, \omega)$ and $\tilde{\mathcal{H}}(\mathbf{k}, \omega)$ are performed by the formulas:

$$
\begin{gathered}
\tilde{\mathcal{E}}(\mathbf{k}, \omega)=i \frac{4 \pi}{\varepsilon} \cdot \frac{\left(\left(\omega^{2}-i \omega \gamma\right) \tilde{\iota}(\mathbf{k}, \omega)-\bar{c}^{2}(\mathbf{k}, \tilde{\iota}(\mathbf{k}, \omega)) \mathbf{k}\right)}{(\omega-i \gamma)\left(\omega^{2}-\bar{c}^{2} \mathbf{k}^{2}-i \omega \gamma\right)} \\
\tilde{\mathcal{H}}(\mathbf{k}, \omega)=-i \frac{4 \pi c}{\varepsilon \mu} \cdot \frac{[\mathbf{k}, \tilde{\iota}(\mathbf{k}, \omega)]}{\left(\omega^{2}-\bar{c}^{2} \mathbf{k}^{2}-i \omega \gamma\right)}
\end{gathered}
$$

Here, the Fourier images $\tilde{\mathcal{E}}(\mathbf{k}, \omega), \tilde{\mathcal{H}}(\mathbf{k}, \omega)$ are generalized functions. 


\section{Energy flux density at the stationary regime}

Let us calculate the average value of the energy flux density $S_{j}(\mathbf{x}, t), j=1,2,3$ of irradiation using the explicit expressions (19) and (20) of Gaussian random fields $\tilde{\mathcal{E}}(\mathbf{k}, \omega), \tilde{\mathcal{H}}(\mathbf{k}, \omega)$,

$$
S_{j}(\mathbf{x}, t)=\int_{\mathbb{R}^{7}} R_{j}\left(\mathbf{x}-\mathbf{y}_{1}, t-s ; \mathbf{x}-\mathbf{y}_{2}, t-s\right) K\left(\left|\mathbf{y}_{1}-\mathbf{y}_{2}\right|\right) a\left(\mathbf{y}_{1}, s ; T\right) a\left(\mathbf{y}_{2}, s ; T\right) d \mathbf{y}_{1} d \mathbf{y}_{2} d s,
$$

where

$$
\begin{gathered}
R_{j}\left(\mathbf{x}, t ; \mathbf{x}^{\prime}, t^{\prime}\right)=\frac{1}{(2 \pi)^{8}} \int_{\mathbb{R}^{8}} \bar{R}_{j}\left(\mathbf{k}, \omega ; \mathbf{k}^{\prime}, \omega^{\prime}\right) \exp \left[i\left((\mathbf{k}, \mathbf{x})-\left(\mathbf{k}^{\prime}, \mathbf{x}^{\prime}\right)\right)+i\left(\omega t-\omega^{\prime} t^{\prime}\right)\right] d \mathbf{k} d \mathbf{k}^{\prime} d \omega d \omega^{\prime} \\
\bar{R}_{j}\left(\mathbf{k}, \omega ; \mathbf{k}^{\prime}, \omega^{\prime}\right)=-R \frac{\left(k_{j}^{\prime}\left(2 \omega(\omega-i \gamma)-\bar{c}^{2} \mathbf{k}^{2}\right)+\bar{c}^{2} k_{j}\left(k_{m} k_{m}^{\prime}\right)\right)}{(\omega-i \gamma)\left(\omega^{2}-\bar{c}^{2} \mathbf{k}^{2}-i \omega \gamma\right)\left(\omega^{\prime 2}-\bar{c}^{2} \mathbf{k}^{\prime 2}+i \omega^{\prime} \gamma\right)} \\
R=4 \pi \bar{c}^{2} / \varepsilon
\end{gathered}
$$

The transformation of the expression (22) on the basis of Eq.(23) leads to the following formula:

$$
S_{j}(\mathbf{x}, t)=S_{j}^{(u)}(\mathbf{x}, t)+S_{j}^{(v)}(\mathbf{x}, t)+S_{j}^{(w)}(\mathbf{x}, t),
$$

where each of performed terms has the form:

$$
\begin{aligned}
& S_{j}^{(u)}(\mathbf{x}, t)=-i R \int_{\mathbb{R}^{7}} U\left(\mathbf{x}-\mathbf{y}_{1}, t-s\right) \nabla_{j} V^{*}\left(\mathbf{x}-\mathbf{y}_{2}, t-s\right) \times \\
& \times K\left(\left|\mathbf{y}_{1}-\mathbf{y}_{2}\right|\right) a\left(\mathbf{y}_{1}, s ; T\right) a\left(\mathbf{y}_{2}, s ; T\right) d \mathbf{y}_{1} d \mathbf{y}_{2} d s, \\
& S_{j}^{(v)}(\mathbf{x}, t)=-R \int_{\mathbb{R}^{7}}\left[\dot{V}\left(\mathbf{x}-\mathbf{y}_{1}, t-s\right)\right]\left[\nabla_{j} V^{*}\left(\mathbf{x}-\mathbf{y}_{2}, t-s\right)\right] \times \\
& \times K\left(\left|\mathbf{y}_{1}-\mathbf{y}_{2}\right|\right) a\left(\mathbf{y}_{1}, s ; T\right) a\left(\mathbf{y}_{2}, s ; T\right) d \mathbf{y}_{1} d \mathbf{y}_{2} d s, \\
& S_{j}^{(w)}(\mathbf{x}, t)=i \bar{c}^{2} R \int_{\mathbb{R}^{7}}\left[\nabla_{m} \nabla_{j} W\left(\mathbf{x}-\mathbf{y}_{1}, t-s\right)\right]\left[\nabla_{m} V^{*}\left(\mathbf{x}-\mathbf{y}_{2}, t-s\right)\right] \times \\
& \times K\left(\left|\mathbf{y}_{1}-\mathbf{y}_{2}\right|\right) a\left(\mathbf{y}_{1}, s ; T\right) a\left(\mathbf{y}_{2}, s ; T\right) d \mathbf{y}_{1} d \mathbf{y}_{2} d s,
\end{aligned}
$$

and generalized functions $U(\mathbf{x}, t), V(\mathbf{x}, t), W(\mathbf{x}, t)$ are defined by following integral representations:

$$
\begin{gathered}
U(\mathbf{x}, t)=\frac{1}{(2 \pi)^{4}} \int_{\mathbb{R}^{4}} \frac{\exp (i(\mathbf{k}, \mathbf{x})+i \omega t)}{\omega-i \gamma} d \mathbf{k} d \omega, \\
V(\mathbf{x}, t)=\frac{1}{(2 \pi)^{4}} \int_{\mathbb{R}^{4}} \frac{\exp (i(\mathbf{k}, \mathbf{x})+i \omega t)}{\omega^{2}-\bar{c}^{2} \mathbf{k}^{2}-i \omega \gamma} d \mathbf{k} d \omega, \\
W(\mathbf{x}, t)=\frac{1}{(2 \pi)^{4}} \int_{\mathbb{R}^{4}} \frac{\exp (i(\mathbf{k}, \mathbf{x})+i \omega t)}{(\omega-i \gamma)\left(\omega^{2}-\bar{c}^{2} \mathbf{k}^{2}-i \omega \gamma\right)} d \mathbf{k} d \omega .
\end{gathered}
$$




\section{Asymptotic expressions of generalized functions $U, V, W$.}

Let us find some asymptotic formulas of generalized functions $U(\mathbf{x}, t), V(\mathbf{x}, t), W(\mathbf{x}, t)$ when the small parameter $\gamma L / \bar{c}$ tends to zero. For the generalized function $U$, one can find easily the explicit form

$$
U(\mathbf{x}, t)=i \Theta(t) \delta(\mathbf{x}) e^{-\gamma t},
$$

where $\Theta(\cdot)$ is the Heaviside function. The functions $V(\mathbf{x}, t)$ and $W(\mathbf{x}, t)$ have no such a simple explicit representation. Therefore, we show the main terms of their asymptotic representation at $\gamma L / \bar{c} \rightarrow 0$ :

$$
\begin{aligned}
V(\mathbf{x}, t)= & \frac{\Theta(t)}{4 \pi \bar{c} r} e^{-\gamma t / 2} D_{-}(r, t), \quad D_{ \pm}(r, t)=\delta(r+\bar{c} t) \pm \delta(r-\bar{c} t) \\
W(\mathbf{x}, t)= & -\frac{i \Theta(t)}{4 \pi r \bar{c}^{2}}\left[e^{-\gamma t}-\frac{1}{2} e^{-\gamma t / 2}(\operatorname{sgn}(r+\bar{c} t)+\operatorname{sgn}(r-\bar{c} t)-\right. \\
& \left.\left.-\frac{\gamma}{2 \bar{c}}[|r+\bar{c} t|-|r-\bar{c} t|]\right)-\frac{r}{2 \bar{c} t}(\pi \gamma t)^{-1 / 2}\right],
\end{aligned}
$$

\section{Integral representations $S_{j}^{(u)}(\mathbf{x}, t), S_{j}^{(v)}(\mathbf{x}, t), S_{j}^{(w)}(\mathbf{x}, t)$.}

Using the asymptotic formula (33), we find integral representations of the functions $S_{j}^{(u)}(\mathbf{x}, t)$, $S_{j}^{(v)}(\mathbf{x}, t), S_{j}^{(w)}(\mathbf{x}, t)$ which do not contain $\delta$-function singularity when $t>0$

$$
\begin{gathered}
V(\mathbf{x}, t)=\frac{\Theta(t)}{4 \pi \bar{c} r} e^{-\gamma t / 2} \delta(r-\bar{c} t) \\
S_{j}^{(u)}(\mathbf{x}, t)=i \frac{R}{4 \pi \bar{c}^{2}} \int_{\mathbb{R}^{6}} \frac{e^{-\gamma\left|\mathbf{y}_{2}\right| / 2 \bar{c}}}{\left|\mathbf{y}_{2}\right|} \nabla_{j}^{(2)}\left[K\left(\left|\mathbf{y}_{2}-\mathbf{y}_{1}\right|\right) a\left(\mathbf{x}-\mathbf{y}_{1}, t-s ; T\right) a\left(\mathbf{x}-\mathbf{y}_{2}, t-s ; T\right)\right]_{s=\left|\mathbf{y}_{2}\right| / \bar{c}} \\
\times U\left(\mathbf{y}_{1},\left|\mathbf{y}_{2}\right| / \bar{c}\right) d \mathbf{y}_{1} d \mathbf{y}_{2}, \\
S_{j}^{(v)}(\mathbf{x}, t)=\frac{R}{4 \pi \bar{c}^{2}} \int_{\mathbb{R}^{6}} \frac{e^{-\gamma\left|\mathbf{y}_{2}\right| / 2 \bar{c}}}{\left|\mathbf{y}_{2}\right|} \nabla_{j}^{(2)}\left[K\left(\left|\mathbf{y}_{2}-\mathbf{y}_{1}\right|\right) a\left(\mathbf{x}-\mathbf{y}_{1}, t-s ; T\right) a\left(\mathbf{x}-\mathbf{y}_{2}, t-s ; T\right)\right]_{s=\left|\mathbf{y}_{2}\right| / \bar{c}} \\
\times \dot{V}\left(\mathbf{y}_{1},\left|\mathbf{y}_{2}\right| / \bar{c}\right) d \mathbf{y}_{1} d \mathbf{y}_{2}, \\
S_{j}^{(w)}(\mathbf{x}, t)=-\frac{i R}{4 \pi} \int_{\mathbb{R}^{6}} \frac{e^{-\gamma\left|\mathbf{y}_{2}\right| / 2 \bar{c}}}{\left|\mathbf{y}_{2}\right|} \nabla_{j}^{(1)} \nabla_{m}^{(1)} \nabla_{m}^{(2)}\left[K\left(\left|\mathbf{y}_{2}-\mathbf{y}_{1}\right|\right) a\left(\mathbf{x}-\mathbf{y}_{1}, t-s ; T\right) a\left(\mathbf{x}-\mathbf{y}_{2}, t-s ; T\right)\right]_{s=\left|\mathbf{y}_{2}\right| / \bar{c}} \\
\times W\left(\mathbf{y}_{1},\left|\mathbf{y}_{2}\right| / \bar{c}\right) d \mathbf{y}_{1} d \mathbf{y}_{2} .
\end{gathered}
$$

To obtain the asymptotic behavior of integrals in Eqs.(36-38) when $x / L \bar{c} \kappa \rightarrow 0$, we assume that the temperature distribution $T(\mathbf{x}, t)$ has the following asymptotic $T(\mathbf{x}, t-s)=T(\mathbf{x}, t)\left(1+\varkappa s O(1) / \kappa L^{2}\right)$ due to Eq.(1). Then, if the limit transition is applied to the integrals (36-38), we need to neglect the temporal shifts in the functions $a(\mathbf{x}, t-s)=a(T(\mathbf{x}, t-s))$, since

$$
a(\mathbf{x}, t-s ; T)=a(\mathbf{x}, t ; T)+\left(\frac{d a(T)}{d T}\right)_{T(\mathbf{x}, t)}(T(\mathbf{x}, t-s)-T(\mathbf{x}, t))+o\left(\frac{\varkappa}{\kappa L \bar{c}}\right) .
$$

As a result, we obtain from formulas (36-38) the following integral representations which permit to calculate the pointed out densities after substitution of the correlation function in the form $K(|\mathbf{x}|)=$ $r_{0}^{-3} Q\left(\mathrm{x}^{2} / 2 r_{0}^{2}\right)$. It is done for the purpose to introduce their dependence on $r_{0}$ in the explicit form:

$$
S_{j}^{(u)}(\mathbf{x}, t)=i \frac{r_{0} R}{4 \pi \bar{c}^{2}} \int_{\mathbb{R}^{6}} \frac{e^{-\gamma r_{0}\left|\mathbf{y}_{2}\right| / 2 \bar{c}}}{\left|\mathbf{y}_{2}\right|}\left[\nabla_{j}^{(2)} Q\left(\left(\mathbf{y}_{2}-\mathbf{y}_{1}\right)^{2} / 2\right) a\left(\mathbf{x}-r_{0} \mathbf{y}_{2}, t ; T\right) U\left(r_{0} \mathbf{y}_{1}, r_{0}\left|\mathbf{y}_{2}\right| / \bar{c}\right)\right]
$$




$$
\begin{gathered}
\times a\left(\mathbf{x}-r_{0} \mathbf{y}_{1}, t ; T\right) d \mathbf{y}_{1} d \mathbf{y}_{2}, \\
S_{j}^{(v)}(\mathbf{x}, t)=\frac{r_{0} R}{4 \pi \bar{c}^{2}} \int_{\mathbb{R}^{6}} \frac{e^{-\gamma r_{0}\left|\mathbf{y}_{2}\right| / 2 \bar{c}}}{\left|\mathbf{y}_{2}\right|}\left[\nabla_{j}^{(2)} Q\left(\left(\mathbf{y}_{2}-\mathbf{y}_{1}\right)^{2} / 2\right) a\left(\mathbf{x}-r_{0} \mathbf{y}_{2}, t ; T\right) \dot{V}\left(r_{0} \mathbf{y}_{1}, r_{0}\left|\mathbf{y}_{2}\right| / \bar{c}\right)\right] \\
\times a\left(\mathbf{x}-r_{0} \mathbf{y}_{1}, t ; T\right) d \mathbf{y}_{1} d \mathbf{y}_{2}, \\
S_{j}^{(w)}(\mathbf{x}, t)=\frac{i R}{4 \pi r_{0}} \int_{\mathbb{R}^{6}} \frac{e^{-\gamma r_{0}\left|\mathbf{y}_{2}\right| / 2 \bar{c}}}{\left|\mathbf{y}_{2}\right|}\left[\nabla_{j}^{(1)} \Delta^{(1)} Q\left(\left(\mathbf{y}_{2}-\mathbf{y}_{1}\right)^{2} / 2\right) a\left(\mathbf{x}-r_{0} \mathbf{y}_{1}, t ; T\right) W\left(r_{0} \mathbf{y}_{1}, r_{0}\left|\mathbf{y}_{2}\right| / \bar{c}\right)\right] \\
\times a\left(\mathbf{x}-r_{0} \mathbf{y}_{2}, t ; T\right) d \mathbf{y}_{1} d \mathbf{y}_{2} .
\end{gathered}
$$

\section{The asymptotic expression of $S_{j}(\mathbf{x}, t)$ at the limit $r_{0} / L \rightarrow 0$.}

Passing to the limit $r_{0} \rightarrow 0$, we calculate main terms of asymptotic expressions of densities $S_{j}^{(u)}(\mathbf{x}, t)$, $S_{j}^{(v)}(\mathbf{x}, t), S_{j}^{(w)}(\mathbf{x}, t)$, using the asymptotic expressions of functions $U(\mathbf{x}, t), V(\mathbf{x}, t), W(\mathbf{x}, t)$, respectively. The main terms are obtained when the amplitude $a(\mathbf{x}, t ; T)$ as well as the asymptotic functions $U(\mathbf{x}, t)$, $V(\mathbf{x}, t), W(\mathbf{x}, t)$ in subintegral expressions are not differentiated on spatial arguments. As the result, we obtain the asymptotic formula

$$
S_{j}^{(u)}=-a^{2}(\mathbf{x}, t ; T)\left(\frac{r_{0}^{-2} R}{4 \pi \bar{c}^{2}}\right) \int_{\mathbb{R}^{3}} \frac{\nabla_{j} Q\left(\mathbf{y}^{2} / 2\right)}{|\mathbf{y}|} d \mathbf{y}+r_{0}^{-2} o(1),
$$

where the integral is equal to zero due to the spherical symmetry of the correlation function. Finally, we have $S_{j}^{(u)}=r_{0}^{-2} o(1)$ when $r_{0} \rightarrow 0$.

We shall calculate the asymptotic of the integral $S_{j}^{(v)}(\mathbf{x}, t)$ using the function $V(\mathbf{x}, t)$ asymptotic. At the same time, the temporal derivative of this asymptotic is equal to

$$
\dot{V}(\mathbf{x}, t)=-\frac{\gamma}{2} V(\mathbf{x}, t)-\frac{\Theta(t)}{4 \pi r} e^{-\gamma t / 2} \delta^{\prime}(r-\bar{c} t)
$$

when $t>0$. Substitution of this expression and transformation of the subintegral expression leads to the following final result:

$$
S_{j}^{(v)}(\mathbf{x}, t)=\frac{R Q_{0}}{8 \pi \bar{c}^{2}} \int_{\mathbb{R}^{3}} \frac{y_{j} e^{-\gamma|\mathbf{y}| / \bar{c}}}{|\mathbf{y}|^{3}} a^{2}(\mathbf{x}-\mathbf{y}, t ; T) d \mathbf{y} .
$$

This formula shows that functions $S_{j}^{(u)}(\mathbf{x}, t), S_{j}^{(v)}(\mathbf{x}, t)$ may be neglected when the main asymptotic term $S_{j}(\mathbf{x}, t)$ is calculated.

Substituting the asymptotic expression (34) into Eq.(41) and using the change of integration variables $r_{0} \mathbf{y}_{j} \Rightarrow \mathbf{y}_{j}, j=1,2$, we find

$$
\begin{gathered}
S_{j}^{(w)}(\mathbf{x}, t)=\frac{r_{0}^{-3} R}{(4 \pi \bar{c})^{2}} \int_{\mathbb{R}^{6}} \frac{e^{-\gamma\left|\mathbf{y}_{2}\right| / 2 \bar{c}}}{\left|\mathbf{y}_{2}\right|\left|\mathbf{y}_{1}\right|}\left[\nabla_{j}^{(1)} \Delta^{(1)} Q\left(\left(\mathbf{y}_{2}-\mathbf{y}_{1}\right)^{2} / 2 r_{0}^{2}\right)\right] a\left(\mathbf{x}-\mathbf{y}_{1}, t ; T\right) a\left(\mathbf{x}-\mathbf{y}_{2}, t ; T\right) \times \\
\times\left[e^{-\gamma\left|\mathbf{y}_{2}\right| / \bar{c}}+\frac{1}{2} e^{-\gamma\left|\mathbf{y}_{2}\right| / 2 \bar{c}}\left(1+\operatorname{sgn}\left(\left|\mathbf{y}_{1}\right|-\left|\mathbf{y}_{2}\right|\right)-\frac{\gamma}{2 \bar{c}}\left[\left|\mathbf{y}_{1}\right|+\left|\mathbf{y}_{2}\right|-|| \mathbf{y}_{1}|-| \mathbf{y}_{2}||\right]\right)-\right. \\
\left.-\frac{\left|\mathbf{y}_{1}\right|}{2\left|\mathbf{y}_{2}\right|^{3 / 2}}\left(\frac{\bar{c}}{\pi \gamma}\right)^{1 / 2}\right] d \mathbf{y}_{1} d \mathbf{y}_{2} .
\end{gathered}
$$

It is easy to show that the last term does not lead to the faster asymptotic when $r_{0} \rightarrow 0$. It tends to a finite limit in the mentioned case. Similarly, we may neglect the next-to-last term in the subintegral 
expression (45). Thus, it is necessary to calculate the asymptotic associated with the first two terms. The coefficient at the asymptotic term proportional to $r_{0}^{-3}$ of the first summand

$$
\begin{array}{r}
S_{j}^{(w, 1)}(\mathbf{x}, t) \equiv \frac{r_{0}^{-3} R}{(4 \pi \bar{c})^{2}} \int_{\mathbb{R}^{6}} \frac{e^{-3 \gamma\left|\mathbf{y}_{2}\right| / 2 \bar{c}}}{\left|\mathbf{y}_{2}\right|\left|\mathbf{y}_{1}\right|}\left[\nabla_{j}^{(1)} \Delta^{(1)} Q\left(\left(\mathbf{y}_{2}-\mathbf{y}_{1}\right)^{2} / 2 r_{0}^{2}\right)\right] \times \\
\times a\left(\mathbf{x}-\mathbf{y}_{1}, t ; T\right) a\left(\mathbf{x}-\mathbf{y}_{2}, t ; T\right) d \mathbf{y}_{1} d \mathbf{y}_{2}
\end{array}
$$

is equal to zero.

Thus, the main asymptotic term of the function $S_{j}^{(w)}(\mathbf{x}, t)$ is determined by the following summand

$$
\begin{aligned}
S_{j}^{(w, 2)}(\mathbf{x}, t) \equiv \frac{r_{0}^{-3} R}{(4 \pi \bar{c})^{2}} \int_{\mathbb{R}^{6}:\left|\mathbf{y}_{1}\right|>\left|\mathbf{y}_{2}\right|} \frac{e^{-\gamma\left|\mathbf{y}_{2}\right| / \bar{c}}}{\left|\mathbf{y}_{2}\right|\left|\mathbf{y}_{1}\right|}[ & \left.\nabla_{j}^{(1)} \Delta^{(1)} Q\left(\left(\mathbf{y}_{2}-\mathbf{y}_{1}\right)^{2} / 2 r_{0}^{2}\right)\right] \times \\
& \times a\left(\mathbf{x}-\mathbf{y}_{1}, t ; T\right) a\left(\mathbf{x}-\mathbf{y}_{2}, t ; T\right) d \mathbf{y}_{1} d \mathbf{y}_{2} .
\end{aligned}
$$

Making the same changes of integration variables when we have done at the analysis of the function $S_{j}^{(w, 1)}(\mathbf{x}, t)$ and passing to the limit $r_{0} \rightarrow 0$, we obtain the main asymptotic term of analyzed function in the following form:

$$
S_{j}^{(w)}(\mathbf{x}, t) \sim S_{j}^{(w, 2)}(\mathbf{x}, t)=\frac{r_{0}^{-3} R Q_{0}}{8 \pi \bar{c}^{2}} \int_{\mathbb{R}^{3}} \frac{y_{j} e^{-\gamma \mid \mathbf{y} / \bar{c}}}{|\mathbf{y}|^{3}} a^{2}(\mathbf{x}-\mathbf{y}, t ; T) d \mathbf{y} .
$$

\section{Conclusion.}

The expression (46) which has the main order as $r_{0} \rightarrow 0$, gives final expression of the energy flux density asymptotic of the fluctuating electromagnetic field

$$
S_{j}(\mathbf{x}, t)=\frac{r_{0}^{-3} R Q_{0}}{4 \pi \bar{c}^{2}} \int_{\mathbb{R}^{3}} \frac{(\mathbf{x}-\mathbf{y})_{j} e^{-\gamma|\mathbf{x}-\mathbf{y}| / \bar{c}}}{|\mathbf{x}-\mathbf{y}|^{3}} a^{2}(\mathbf{y}, t ; T) d \mathbf{y},
$$

where the function $a^{2}(\mathrm{x}, t ; T)$ is given by the expression (5). It solves the problem formulated at the beginning of present work.

The derived expression of the energy flux density may be obtained in principal on the basis of arguments used in the theory of radiation transfer in medium (see [1]-[3]). However, we must to improve these arguments substantially. Such a modification is connected with the fact that there is the significant difference from the formula of the standard theory. In frameworks of radiation transfer theory, the energy flux density is derived on the basis of geometric optics presentation applying them for physical situation that is under study at present work. Then, the obtained formula differs from Eq.(47) by the supplement multiply $|\mathbf{y}|^{-2}$ in integral kernel. The appearance of this weight shows that the Beer-Bouguer-Lambert law which describes the passage of radiation through medium, is not completely true for thermal radiation generated by the medium and it is spread in it. The appearance of the modification may be understood from the general physics view point. Besides damping of the radiation is obeying to the Beer-BouguerLambert law when it passes thorough the medium, there is also the damping connected with the presence of isotropic «dissipation» of radiation that is emitted by each spatial point when the last come to it. At this, the appearance of the supplement weight $|\mathbf{y}|^{-2}$ in the energy flux density that is irradiated from the point $\mathbf{y}=0$, is connected with the total energy conservation at such a dissipation when the radiation passed the distance $|\mathbf{y}|$. Namely, if we shall not take into account the Beer-Bouguer-Lambert absorption, the total energy flowing through the sphere surface with the square $4 \pi|\mathbf{y}|^{2}$, is constant. Such an effect is not taken into account in frameworks of the standard radiation transfer theory. 


\section{References}

1. E.M. Sparrow, R.D. Cess, Radiation heat transfer, Brooks/Cole Publishing Company, Belmont, California, (1970).

2. N.A. Rubtsov, Radiation heat transfer in continuos media, Nauka, Siber. Department, Novosibirsk (1984) [in Russian].

3. V.A. Petrov, N.V. Marchenko, Radiation energy transfer in partially transparent solid materials, Nauka, Moscow (1985) [in Russian].

4. A.V. Kolesnikov, Yu.P. Virchenko, Functional Materials, 13, 372 (2006).

5. Yu.P. Virchenko, M.A. Saprykin, Scientific Bulletin of Belgorod State University. Mathematics \& Physics, 16, 47 (2009) [in Russian].

6. Yu.P. Virchenko, M.A. Saprykin, Functional Materials, 18, 504 (2011).

7. Yu.P. Virchenko, M.A. Saprykin, Reports of NASU, 12, 63 (2010) [in Russian].

8. S.M. Rytov, Theory of electrical fluctuations and thermal radiation, AN SSSR Publishing, Moscow (1953) [in Russian].

9. S.M. Rytov, Yu.A. Klyatskin, V.I. Tatarskii, Introduction to Statistical Radiophysics. v.2. Random fields, Nauka, Moscow (1978) [in Russian].

10. Yu.P. Virchenko, Lam Tan Phat, Functional Materials, 23, 075 (2016). 